\title{
ORIGINAL ARTICLE \\ The consequences of genetic variation in sex peptide expression levels for egg laying and retention in females
}

\author{
DT Smith ${ }^{1}$, LK Sirot ${ }^{2,3}$, MF Wolfner ${ }^{3}$, DJ Hosken ${ }^{1}$ and N Wedell ${ }^{1}$
}

The accessory gland proteins (Acps) that male Drosophila melanogaster produce and transfer to females during copulation are key to male and female fitness. One Acp, the sex peptide (SP), is largely responsible for a dramatic increase in female egg laying and decrease in female receptivity after copulation. While genetic variation in male $S P$ expression levels correlate with refractory period duration in females, it is unknown whether male $S P$ expression influences female egg laying or if any effect of SP is mediated by SP retention in the female reproductive tract. Here we measured the amount of SP retained in the female reproductive tract after mating and female egg laying after copulating with virgin males. We found no correlation between male $S P$ expression levels and egg laying, or the amount of SP in the female reproductive tract after mating. Additionally, the amount of SP retained in the female did not influence egg laying. These finding suggests that additional factors, such as variation in other Acps, are important for the retention of SP in females and its quantitative effects on egg laying. It also shows that egg laying and refractory period response to SP is at least partially uncoupled.

Heredity (2012) 109, 222-225; doi:10.1038/hdy.2012.32; published online 13 June 2012

Keywords: Drosophila melanogaster; sex peptide; sexual conflict; sperm competition

\section{INTRODUCTION}

For female insects, mating often induces large transcriptional, physiological and behavioural changes that are often triggered by components of male seminal fluid (Avila et al., 2011). These changes and the molecules that cause them have been studied in detail in Drosophila melanogaster (Findlay et al., 2008). In this species, over 138 seminal proteins and peptides are transferred (along with sperm) to females during mating (Avila et al., 2011). These seminal fluid components are important for male and female fitness and, among other functions, they facilitate successful sperm storage (Adams and Wolfner, 2007), influence the outcome of sperm competition (Begun et al., 2000; Chapman et al., 2001), reduce female receptivity (Chapman et al., 2003; Liu and Kubli, 2003) and stimulate ovulation (Heifetz et al., 2000; Chapman et al., 2001).

The function of one accessory gland protein (Acp), in particular, the sex peptide (SP) has been well-studied in D. melanogaster. When injected into (Chen et al., 1988) or ectopically expressed (Aigaki et al., 1991) in females, SP causes several profound changes in female behaviour. Two of the most striking of these are increased egg laying and reduced acceptance of male-mating attempts (Chapman et al., 2003; Liu and Kubli, 2003). These are caused by the sex-peptide binding to a neuronal sex peptide receptor (SPR) in the female (Yapici et al., 2008; Hasemeyer et al., 2009; Yang et al., 2009).

In D. melanogaster there is sequence variation within the SP gene and in upstream regions (Cirera and Aguade, 1997), which could potentially influence the regulation of SP. Smith et al. (2009) found variation in $S P$ transcript abundance between genetic isolines of D. melanogaster, suggesting that transcriptional regulatory elements may be important for the natural variation of $S P$ expression. There is also evidence that variation in SP expression influences male fitness, with males that produce relatively high and relatively low amounts of SP RNA inducing the longest refractory periods in females (Smith et al., 2009), which suggests that SP transcript levels is subject to disruptive selection. As the genetic variation of SP expression levels in males have been previously attributed to a quadratic relationship with female refractory period, we expect this same relationship to occur with female egg laying, a behaviour also attributed to SP. An alternative prediction is that SP acts on egg laying via a different mechanism to its effect on refractory period. This possibility remains to be tested. It is therefore important to determine if $S P$ expression levels correlate with the egg-laying response by females, and if any potential relationship may be explained by the amount of SP retained by females after mating.

In this study, we tested whether male isoline $S P$ expression levels or SP retention correlated with the number of eggs laid by females. To further investigate the mechanisms involved in this pathway, we also tested if SP expression levels correlated with the amount of SP retained in females after mating.

\section{MATERIALS AND METHODS}

Fly lines and quantitative real time polymerase chain reaction We maintained all flies and measured SP expression levels as in Smith et al. (2009) using a subset of the inbred lines used in Ayroles et al. (2009). We used isoline numbers $75,85,159,195,345,349,378,449,535,701$ and 804 , which were kindly donated to us by Frank Jiggins (University of Cambridge). We kept isolines on standard food at $25^{\circ} \mathrm{C}$ with a 12:12-h light:dark-light cycle. We diagnosed the isolines for the presence of Wolbachia infection following

${ }^{1}$ Centre for Ecology and Conservation, Biosciences, College of Life \& Environmental Sciences, University of Exeter, Penryn, Cornwall, UK; ${ }^{2}$ Biology, The College of Wooster, Wooster, OH, USA and ${ }^{3}$ Department of Molecular Biology and Genetics, Cornell University, Ithaca, NY, USA

Correspondence: Professor N Wedell, Centre for Ecology and Conservation, Biosciences, College of Life \& Environmental Sciences, University of Exeter, Cornwall Campus, Penryn, Cornwall TR10 9EZ, UK.

E-mail: N.Wedell@exeter.ac.uk

Received 8 December 2011; revised 21 March 2012; accepted 1 May 2012; published online 13 June 2012 
methods in Snook et al. (2000). We reared experimental flies in standardised conditions by placing 40 eggs on $\sim 7 \mathrm{ml}$ of food. As Wolbachia infection is known to influence several aspects of reproductive behaviour, we tested for its influence and found no effect (Smith, 2010). Thus, we refrain from any further discussion of effects of Wolbachia. We collected virgin males from the standardised egg-density vials and kept them individually with food before all experiments. For $S P$ expression analysis, we snap froze 5-day-old males in liquid nitrogen and extracted total RNA (using Tri reagent (Sigma, St Louis, MO, USA)) to measure SP RNA levels with FullVelocity SYBR Green one-step Q-RTPCR Reagents (Stratagene, Santa Clara, CA, USA) with SP-specific primers (Smith et al., 2009). We used the housekeeping gene RP49 to normalise expression levels.

\section{Egg laying}

We measured female egg laying after mating to males from the isolines of interest. To minimise unexpected individual differences amongst males within and between isolines, all males were reared individually to 5 days of age before experiments. To minimise individual differences amongst females of the standard isoline, females were collected in the same fashion as the experimental males but housed in groups of 20 before experimentation. We placed females without anaesthesia with individual virgin males from experimental isolines and observed copulation. We used 10 isolines with between 5 and 13 males per isoline. Immediately after the end of mating, we placed individual females in $9 \times 2.5 \mathrm{~cm}$ universal tubes with a $2 \times 1 \mathrm{~cm}$ laying cap containing standard food with a small amount of red food dye to increase the visibility of eggs laid on the food. Laying caps were replaced and eggs counted daily for 10 days. For analysis, we split the number of eggs laid by each female into two groups. Initially, we assessed the total number of eggs laid after 3 days, as this is when SP has its greatest effect on egg laying (Liu and Kubli, 2003). We also assessed the total number of eggs laid after a 10-day period. Previous work on Drosophila has shown that short-term measures (7 days) of reproductive output is significantly correlated with lifetime reproductive success (Taylor et al., 2008), therefore 10 days of egg laying should be correlated with long-term reproductive output.

\section{$S P$ retention in females}

For the measurement of SP retention in the female reproductive tract after mating, we conducted all matings with males from 11 of the isolines quantified for SP expression levels (Smith et al., 2009). We reared males as described above and we used individual virgin females from a standard isoline, which was independent from the male isolines. Twenty-four hour before experiments we placed individual females in a single vial with food without live yeast. On the day of mating, we aspirated individual males (without anaesthesia) into a vial containing a single female and observed mating. One hour after each female had finished copulation, we snap froze females in liquid nitrogen and stored them at $-80^{\circ} \mathrm{C}$.

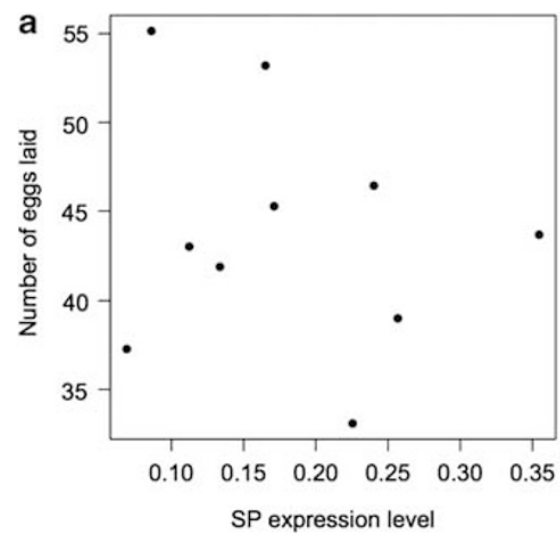

Figure 1 The relationship between the mean cumulative number of eggs laid in (a) 3 or (b) 10 days after mating and the mean SP expression levels of males from different isolines $(n=10)$. Results were the same when using line median or rank values (data not shown).

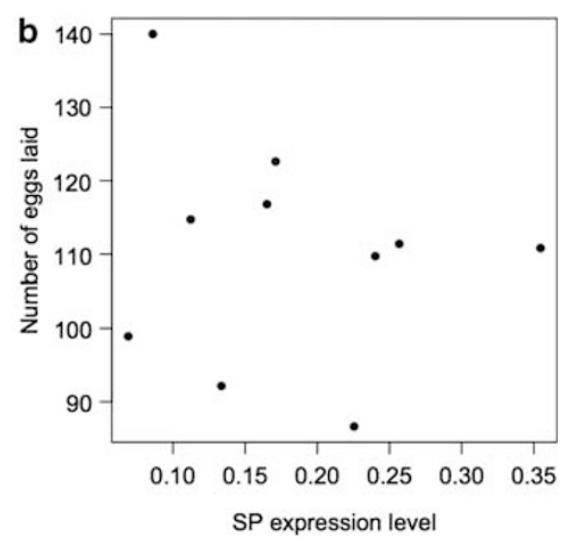

Following Sirot et al. (2009) and Wigby et al. (2009) with minor modifications, we used the ELISA technique to determine how much SP was present in the female reproductive tract after mating. Briefly, we dissected and homogenised the lower female reproductive tract in 10\% Dulbecco's Phosphate Buffering Solution with protease inhibitor (Roche Complete Protease Inhibitor tablets, Indianapolis, IN, USA). We used ELISA plates (BD Biosciences, San Jose, CA, USA) with 1:2000 dilution of primary antiSP antibody (kindly provided by E. Kubli (Peng et al., 2005)) to bind to SP present in the samples and 1:2000 diluted horseradish peroxidase-conjugated goat antirabbit secondary antibody (Jackson ImmunoResearch Laboratories, Inc., West Grove, PA, USA) to react with $3,3^{\prime}, 5,5^{\prime}$-tetramethylbenzidine substrate (KPL, Gaithersburg, MD, USA) in a colourimetric reaction. We then measured the optical density (OD) at $\lambda=450 \mathrm{~nm}\left(\mathrm{OD}_{450}\right)$. For standardisation, we included serial dilutions of protein from the equivalent of the accessory glands from a single Canton-S fly male on each plate of ELISA reactions.

\section{Statistical analysis}

We conducted all statistical analysis using $\mathrm{R}$ for Macintosh version 2.9.2 (R Development Core Team, 2009). We conducted analysis on male isoline mean and individual values. Isoline means approximate genotypic associations (David et al., 2005) and individual values approximates the phenotypic associations. Females that did not lay any eggs were removed from analysis for the egg-laying assay $(n=5)$. Results using isoline means and medians showed the same relationships, so here we only present results using isoline means.

To examine the impact of variation in isoline mean $S P$ transcript levels on egg laying, we regressed isoline mean $S P$ expression level values, including the quadratic term, against the number of eggs laid early (over 3 days) or in total (over 10 days) by females after mating to males from our experimental isolines using least-squared regression. We included mean female size as a covariate. We also regressed individual female egg laying against individual female size to test if larger female lay more eggs, a pattern that is expected for this species (Lefranc and Bundgaard, 2000) and hence a test of our egg-laying assay.

We analysed the relationship between SP retention and egg laying using least-squared regression with eggs laid either 3 or 10 days after mating as the response variable and SP retention and its quadratic term as the explanatory variables and female size as a covariate. We used least-squared regression to investigate the relationship between SP expression levels and SP retention in a similar manner.

\section{RESULTS}

We found no relationship between mean isoline SP expression levels and the number of eggs laid by females either $3\left(\mathrm{~F}_{(1,6)}=0.08\right.$, $P=0.72)$ or 10 days after mating $\left(\mathrm{F}_{(1,6)}=0.27, P=0.62\right)$ (Figure 1). Smith et al. (2009) previously showed a quadratic relationship between male $S P$ expression levels and refractory period duration in females and therefore we considered such a relationship. As seen on Figure 1, there was no quadratic relationship between $S P$ 

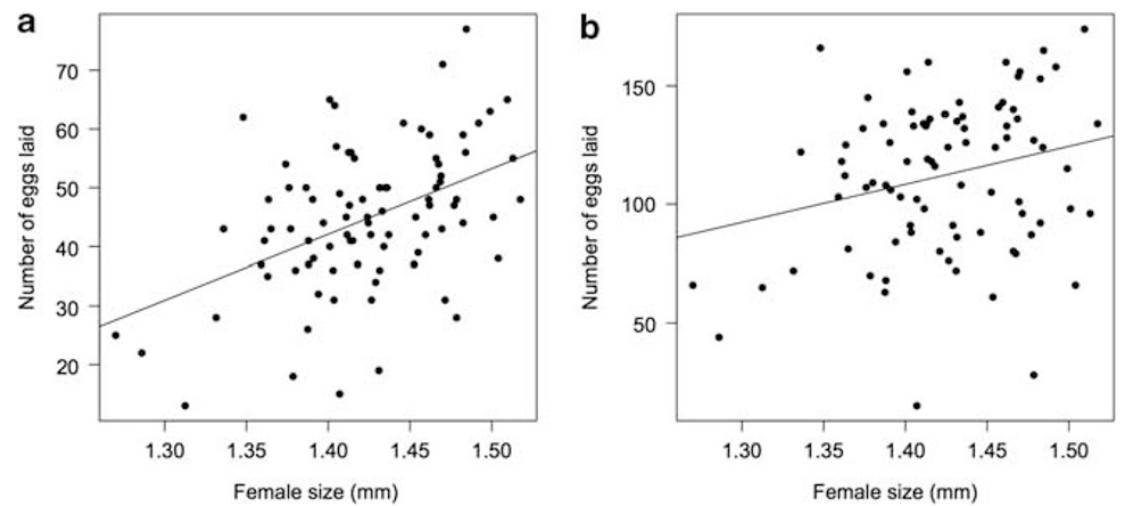

Figure 2 Female size is positively correlated with the cumulative number of eggs laid (a) 3 and (b) 10 days after mating. Five females did not lay any eggs and were not included in this analysis $(n=86)$.
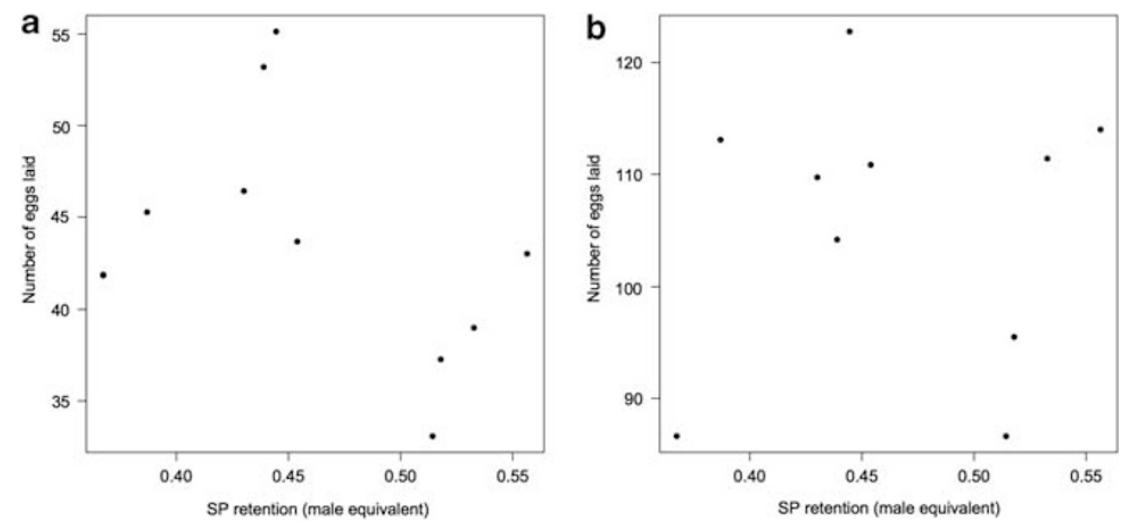

Figure 3 The relationship between the mean cumulative number of eggs laid in (a) 3 or (b) 10 days after mating and the mean SP retention of males from different isolines $(n=10)$. Results were similar when using line median or rank values (data not shown).

expression and egg laying after 3 days egg laying $\left(\mathrm{F}_{(1,6)}=0.38\right.$, $P=0.56)$ or 10 days egg laying $\left(\mathrm{F}_{(1,6)}=0.14, P=0.72\right)$. The mean size of females mated to males from each isoline was not significantly associated with number of eggs laid $\left(\mathrm{F}_{(1,6)}=4.77, P=0.072\right.$ and $\mathrm{F}_{(1,6)}=1.07, P=0.34$ for cumulative eggs laid 3 and 10 days after mating, respectively). There was a significant relationship between the number of eggs laid $3\left(\mathrm{~F}_{(1,84)}=22.27, R^{2}=0.21, t=4.72, P<0.0001\right)$ and 10 days $\left(\mathrm{F}_{(1,84)}=5.63, R^{2}=0.06, t=2.372, P=0.02\right)$ after mating when we used the individual female size and individual female egg laying in the model instead of line means (Figure 2).

We found no correlation between the isoline mean SP retention and isoline mean number of eggs laid after $3\left(\mathrm{~F}_{(1,6)}=2.48, P=0.17\right)$ or 10 days $\left(\mathrm{F}_{(1,6)}=0.03, P=0.88\right)$. The quadratic term for isoline mean SP retention did also not significantly influence egg laying after $3\left(\mathrm{~F}_{(1,6)}=1.50, P=0.27\right)$ or 10 days $\left(\mathrm{F}_{(1,6)}=0.43, P=0.54\right.$, Figure 3$)$. Isoline mean female size did also not significantly influence egg laying $3\left(\mathrm{~F}_{(1,6)}=1.87, P=0.22\right)$ or 10 days after mating $\left(\mathrm{F}_{(1,6)}=0.01\right.$, $P=0.93)$. There was no significant correlation between isoline mean SP expression levels and isoline mean SP retention $\left(\mathrm{F}_{(1,8)}=0.0001\right.$, $P=0.99$ or the its quadratic term $\left(\mathrm{F}_{(1,8)}=0.14, P=0.72\right.$, Figure 4$)$.

\section{DISCUSSION}

The focus of this study was threefold. First, we investigated whether $S P$ expression levels of different male genotypes correlated with an important fitness correlate influenced by SP, the number of eggs laid by their mates after copulation. We found no correlation between the $S P$ expression levels of male genotypes (as reported in Smith et al.

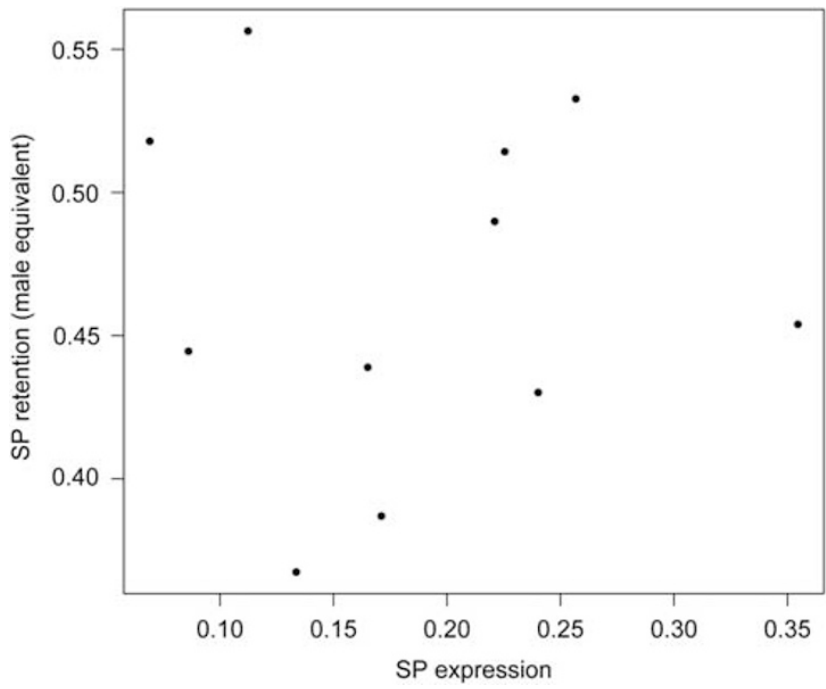

Figure 4 The relationship between isoline mean $S P$ expression levels and isoline mean SP retention in female after mating to a virgin male $(n=11)$.

2009) and the number of eggs laid by their mate after copulation. This is surprising given that previously we showed that genetic variation in the expression levels of SP among isolines of D. melanogaster males correlated with female propensity to remate in a manner suggestive of disruptive selection (Smith et al., 2009). A possible explanation for the 
lack of relationship between SP RNA expression levels and egg laying is that the amount of SP in the female after mating is not reflective by the SP transcript levels. Factors such as translational efficiency of SP RNA, transfer of SP and SP retention could all vary between isolines. It would be of interest to examine the interactions between multiple accessory gland proteins and their fitness effects in the future.

Secondly, to further investigate the potential mechanisms involved in the correlation between SP and egg laying, we tested whether the SP retention of male genotypes were associated with the number of eggs laid by the female that they mated to. We also found no correlation between SP retention and the number of eggs laid by that male's mate after mating. This may be due to effects of other seminal fluid proteins (for example, ovulin, (Heifetz et al., 2000), CG33943 (Ravi Ram and Wolfner, 2007)) that also contribute to the maleinduced effects on egg laying. The fact that refractory period duration if correlated with SP expression levels but that egg laying is not suggests that these two female responses to SP are at least partially uncoupled. Perhaps once SP binds to SPR within the female, these two responses are linked to different neuronal or hormonal pathways.

Thirdly, we tested for a correlation between SP expression levels and SP retention in females. Again, we found no correlation in the SP expression levels in males and the amount of SP that is retained in females after mating. Males can differentially allocate the amount of SP that they transfer to females during copulation (Wigby et al., 2009). SP expression levels may then correlate with the amount of SP (protein) produced by males, yet differential male allocation of SP stocks (that is, not transferring all their stock at once) could potentially mean there is no correlation to $S P$ expression levels and the amount of SP delivered and retained in females. It would be of interest to determine if there is genetic variation in the SP allocation patterns of males among sequential matings and to determine the fitness consequences of this. Additionally, a network of other accessory gland proteins is needed for SP retention in females (Ravi Ram and Wolfner, 2009), and variation in any of the members of that network could potentially affect the amount of SP retained by females. Similarly, variation in amounts of stored sperm could affect retention of SP. SP is actively cleaved from sperm within the female (Monsma et al., 1990), and so interactions between female enzymes that cleave SP (Pilpel et al., 2008) or male enzymes that inhibit enzyme activity (Wolfner et al., 1997) could also dramatically alter the measureable amounts of SP in the female reproductive tract relative to male $S P$ expression levels. Here, we tried to minimise the effect of female variation by using females from the same genetic isoline for all our tests. The lack of correlation between SP expression levels and SP retention in female also suggests that perhaps protein measurements will more accurately reflect relationships between peptides and their pathways than expression levels of genes that code for those peptides.

Overall, these results suggest that SP's effects on refractory period duration and egg laying are at least partially uncoupled. This may be female driven as the pathways influencing the two major effects of SP are unlikely to be identical. These results also suggest that expression patterns do not necessarily reflect the amount of protein that is functioning in the downstream pathways. The use of expression patterns in males may not reflect accurately the true interactions between the sexes.

\section{DATA ARCHIVING}

Data have been deposited at Dryad: doi:10.5061/dryad.77hn0.

\section{CONFLICT OF INTEREST}

The authors declare no conflict of interest.

\section{ACKNOWLEDGEMENTS}

We would like to thank S Wigby, M Hares, N A Buehner for lab support and DTS thanks the Wolfner lab group for their hospitality. This work was supported by the BBSRC (NW), NERC (DJH) and NIH grant ROI-HD038921 (MW).

Adams EM, Wolfner MF (2007). Seminal proteins but not sperm induce morphological changes in the Drosophila melanogaster female reproductive tract during sperm storage. J Insect Physiol 53: 319-331.

Aigaki T, Fleischmann I, Chen PS, Kubli E (1991). Ectopic expression of sex peptide alters reproductive behavior of female $D$. melanogaster. Neuron 7: 557-563.

Avila FW, Sirot LK, LaFlamme BA, Rubinstein CD, Wolfner MF (2011). Insect seminal fluid proteins: identification and function. Annu Rev Entomol 56: 21-40.

Ayroles JF, Carbone MA, Stone EA, Jordan KW, Lyman RF, Magwire MM et al. (2009). Systems genetics of complex traits in Drosophila melanogaster. Nat Genet 41: 299-307.

Begun DJ, Whitley P, Todd BL, Waldrip-Dail HM, Clark AG (2000). Molecular population genetics of male accessory gland proteins in Drosophila. Genetics 156: 1879-1888.

Chapman T, Bangham J, Vinti G, Seifried B, Lung O, Wolfner MF et al. (2003). The sex peptide of Drosophila melanogaster: female post-mating responses analyzed by using RNA interference. Proc Nat Acad Sci USA 100: 9923-9928.

Chapman T, Herndon LA, Heifetz Y, Partridge L, Wolfner MF (2001). The Acp26Aa seminal fluid protein is a modulator of early egg hatchability in Drosophila melanogaster. Proc $R$ Soc Lond B 268: 1647-1654.

Chen PS, Stummzollinger E, Aigaki T, Balmer J, Bienz M, Bohlen P (1988). A male accessory-gland peptide that regulates reproductive behavior of female Drosophila melanogaster. Cell 54: 291-298.

Cirera S, Aguade M (1997). Evolutionary history of the sex-peptide (Acp70A) gene region in Drosophila melanogaster. Genetics 147: 189-197.

David JR, Gibert P, Legout H, Petavy G, Capy P, Moreteau B (2005). Isofemale lines in Drosophila: an empirical approach to quantitative trait analysis in natural populations. Heredity 94: 3-12.

Findlay GD, Yi XH, MacCoss MJ, Swanson WJ (2008). Proteomics reveals novel Drosophila seminal fluid proteins transferred at mating. PLoS Biol 6: 1417-1426.

Hasemeyer M, Yapici N, Heberlein U, Dickson BJ (2009). Sensory neurons in the Drosophila genital tract regulate female reproductive behavior. Neuron 61: 511-518.

Heifetz Y, Lung O, Frongillo EA, Wolfner MF (2000). The Drosophila seminal fluid protein Acp26Aa stimulates release of oocytes by the ovary. Curr Biol 10: 99-102.

Lefranc A, Bundgaard J (2000). The influence of male and female body size on copulation duration and fecundity in Drosophila melanogaster. Hereditas 132: 243-247.

Liu HF, Kubli E (2003). Sex-peptide is the molecular basis of the sperm effect in Drosophila melanogaster. Proc Nat Acad Sci USA 100: 9929-9933.

Monsma SA, Harada HA, Wolfner MF (1990). Synthesis of two Drosophila male accessory gland proteins and their fate after transfer to the female during mating. Dev Biol 142: 465-475.

Peng J, Chen S, Busser S, Liu HF, Honegger T, Kubli E (2005). Gradual release of sperm bound sex-peptide controls female postmating behavior in Drosophila. Curr Biol 15: 207-213.

Pilpel N, Nezer I, Applebaum SW, Helfetz Y (2008). Mating-increases trypsin in female Drosophila hemolymph. Insect Biochem Mol Biol 38: 320-330.

Ravi RK, Wolfner MF (2007). Sustained post-mating response in Drosophila melanogaster requires multiple seminal fluid proteins. PLoS Genet 3: 2428-2438.

Ravi RK, Wolfner MF (2009). A network of interactions among seminal proteins underlies the long-term postmating response in Drosophila. Proc Nat Acad Sci USA 106: 15384-15389.

R Development Core Team (2009). R: a language and environment for statistical computing. R Foundation for Statistical Computing. Vienna: 3-900051-07-0. http:// www.R-project.org.

Sirot LK, Buehner NA, Fiumera AC, Wolfner MF (2009). Seminal fluid protein depletion and replenishment in the fruit fly, Drosophila melanogaster: an ELISA-based method for tracking individual ejaculates. Behav Ecol Sociobiol 63: 1505-1513.

Smith DT (2010). Sex Peptide Evolution and the Impact of Selfish Genetic Elements. PhD Thesis, University of Exeter, UK.

Smith DT, Hosken DJ, ffrench-Constant RH, Wedell N (2009). Variation in sex peptide expression in D. melanogaster. Genet Res 91: 237-242.

Snook RR, Cleland SY, Wolfner MF, Karr TL (2000). Offsetting effects of Wolbachia infection and heat shock on sperm production in Drosophila simulans: analyses of fecundity, fertility and accessory gland proteins. Genetics 155: 167-178.

Taylor ML, Wedell N, Hosken DJ (2008). Sexual selection and female fitness in Drosophila simulans. Behav Ecol Sociobiol 62: 721-728.

Wigby S, Sirot LK, Linklater JR, Buehner N, Calboli FCF, Bretman A et al. (2009). Seminal fluid protein allocation and male reproductive success. Curr Biol 19: 751-757.

Wolfner MF, Harada HA, Bertram MJ, Stelick TJ, Kraus KW, Kalb JM et al. (1997). New genes for male accessory gland proteins in Drosophila melanogaster. Insect Biochem Mol Biol 27: 825-834.

Yang CH, Rumpf S, Xiang Y, Gordon MD, Song W, Jan LY et al. (2009). Control of the postmating behavioral switch in Drosophila females by internal sensory neurons. Neuron 61: 519-526.

Yapici N, Kim YJ, Ribeiro C, Dickson BJ (2008). A receptor that mediates the post-mating switch in Drosophila reproductive behaviour. Nature 451: 33-U1. 